\title{
VALIDACIÓN DEL MÉTODO PARA LA DETERMINACIÓN DE ORO POR ENSAYO AL FUEGO COMBINADO CON ESPECTROMETRÍA DE ABSORCIÓN ATÓMICA EN MUESTRAS GEOLÓGICAS MINERAS METALÚRGICAS
}

\author{
VALIDATION OF THE GOLD DETERMINATION METHOD BY FIRE ASSAY \\ COMBINED WITH ATOMIC ABSORPTION SPECTROMETRY \\ IN GEOLOGICAL, MINE AND METALLURGICAL SAMPLES
}

Diana Buitrón O. ${ }^{1}$ Diego Barona D. ${ }^{1}$ Francisco Iturra M. ${ }^{*}$ \& Johana León F.

Recibido: 14 de agosto 2020 / Aceptado: 14 de diciembre 2020 DOI: 10.26807/ia.v9i1.188

Palabras clave: Absorción atómica; control minero; ensayo al fuego; oro; validación.

Keywords: Atomic absorption; fire assay; gold; mine control; validation.

\section{RESUMEN}

La presente investigación describe el proceso de validación del método para la determinación de oro por ensayo al fuego combinado con espectrofotometría

1 Instituto de Investigación Geológico y Energético, Quito Ecuador (diana.buitron@geoenergía.gob.ec; diego.barona@geoenergía.gob.ec; correspondencia *: francisco.iturra@geoenergía.gob.ec; Johanna.leon@geoenergía.gob.ec). 
de absorción atómica en sedimentos, rocas, concentrados y relaves, con el objetivo de obtener resultados confiables. En el estudio se establecieron los parámetros de validación, los cuales son: límite de detección (LD), límite de cuantificación (LC), intervalo de trabajo, linealidad, selectividad y especificidad, repetibilidad y precisión intermedia, veracidad e incertidumbre, y sus criterios de cumplimiento. Mediante análisis estadístico de los resultados experimentales, y junto a los criterios de aceptación y rechazo, se evaluaron los parámetros de validación antes mencionados. Finalmente, el método implementado por el laboratorio químico del IIGE para la determinación de oro, se declara validado en muestras de sedimentos, rocas, concentrados y relaves.

\section{ABSTRACT}

This investigation describes the process of validation of the method for the determination of gold by fire assay combined with atomic absorption spectrophotometry in sediments, rocks, concentrates and tailings, with the objective of providing reliable results. In the study, the validation parameters were established, which are limit of detection (LOD), limit of quantification (LOQ), working interval, linearity, selectivity and specificity, repeatability and intermediate precision, veracity and uncertainty, and their criteria of compliance. By means of statistical analysis of the experimental results and together with the acceptance and rejection criteria, the validation parameters were evaluated. Finally, the method implemented by the IIGE chemical laboratory for the determination of gold is declared validated in sediment, rock, concentrate and tailings samples.

\section{INTRODUCCIÓN}

La extracción de oro ha sido una actividad económica importante en varias culturas a lo largo de la historia de la humanidad por su belleza, color, resistencia a la corrosión y facilitar la fabricación de objetos admi- 
rables. Alrededor del $51 \%$ del oro es utilizado en joyería y artefactos decorativos, el $34 \%$ es usado en las tiendas financieras del gobierno, lingotes y monedas y alrededor del $12 \%$ es usado en la industria (Laird, 2014).

El oro puede encontrarse en forma nativa o formando compuestos, se conocen alrededor de 20 minerales de oro diferentes $y$, todos son bastante raros. El oro se encuentra en las vetas hidrotermales depositadas por soluciones ascendentes, como partículas diseminadas a través de algunos depósitos de sulfuro, y en depósitos de placer (King, 2019).

El ensayo al fuego es la técnica más ampliamente utilizada y antigua para la determinación del oro, se cree que su uso data del cuarto milenio antes de Cristo. La técnica consta de tres etapas: en la primera se funde la muestra en presencia de varios químicos como el óxido de plomo II (PbO), carbón y otros, para formar una fase metálica con plomo, en la segunda se recupera el oro del plomo, y en la final se realiza la cuantificación del oro (Ghosh et al., 2019). El ensayo al fuego es ampliamente utilizado principalmente por dos razones: se asume que es una técnica que obtiene una recuperación total de del oro y permite utilizar gran cantidad de muestra, lo que vuelve el ensayo más representativo (Wang et al., 2016). Además, permite ser altamente reproducible para todas las matrices geológicas y metalúrgicas, fácilmente adaptable a las condiciones de las minas, costo moderado y resultados rápidos. Finalmente, el ensayo al fuego permite eliminar muchos de los interferentes como sulfuros y óxidos (Ghosh et al., 2019).

En el ensayo al fuego se requieren de varios reactivos. A continuación, se enlistan los más importantes, con sus principales funciones. El óxido de plomo II (PbO), conocido también como litargirio, es utilizado para proveer plomo, para colectar oro y plata (cuando el litargirio se reduce a plomo, se deposita en el fundido por su alto peso específico colectando por afinidad y también por peso específico al oro y plata), el carbonato de sodio $\left(\mathrm{Na}_{2} \mathrm{CO}_{3}\right)$ reduce la temperatura de fusión de la muestra, el bórax $\left(\mathrm{Na}_{2} \mathrm{~B}_{4} \mathrm{O}_{7} 10 \mathrm{H}_{2} \mathrm{O}\right)$ disminuye la temperatura de formación de la escoria, la harina (fuente de carbón) ayuda a la reducción del litargirio a 
plomo elemental (Pb). (Santos et al., 2019). Rodríguez et al. (2018) afirmaron que se puede utilizar entre 10 y 50 g de muestra para el ensayo al fuego.

Uno de los principales parámetros en el ensayo al fuego es el tamaño de partícula de la muestra. Así, cuando variamos el tamaño de partícula de la muestra también varía la concentración recuperada de oro. Los tamaños ideales de partícula de la muestra son los menores o iguales a 0,075 mm (Osman Ali Salih, 2018). En el análisis de oro mediante ensayo al fuego se debe considerar que las recuperaciones de oro cuando está presente el teluro suelen ser bajas (Liu et al.,2019; Santos-Munguía et al., 2019).

Una variante del ensayo al fuego tradicional es el cambio del plomo por níquel, pero se reportan varios problemas y bajas recuperaciones (Liu et al., 2019).

Dentro de las técnicas para determinar oro, luego de su recuperación y purificación, se encuentra la espectroscopia de absorción atómica con Ilama, la espectroscopia de absorción atómica con horno de grafito, y la es- pectrometría de masas con plasma acoplado inductivamente, el requisito para utilizarlas es que el oro se encuentre en solución. Se obtienen con esas técnicas límites de detección en el orden de 20 ng/g, 0,1 ng/g y $0,01 \mathrm{ng} / \mathrm{g}$, respectivamente (Balaram et al., 2012). Otras técnicas que pueden ser utilizadas son la espectrometría de emisión óptica acoplada inductivamente con plasma y la espectrofotometría de absorción ultravioleta y visible (Rodríguez-Rodríguez et al., 2018).

Para la determinación cuantitativa de oro, existen algunos métodos alternativos al ensayo al fuego. Entre ellos están el uso de solventes orgánicos e inorgánicos. En la extracción con solventes inorgánicos se emplea agua regia. Este método se ha utilizado para disolver metales preciosos desde tiempos medievales. La principal mejora moderna es la implementación de equipos más eficientes y automatizados para la digestión de la muestra, como es el uso de hornos microondas o la digestión usando radiación infrarroja enfocada (Wang et al., 2016). 
Por otro lado, los solventes orgánicos más utilizados son diisobutilcetona (DIBC) y metilisobutilcetona (MIBC), después de la disolución completa del oro de la muestra en agua regia. Estos permiten purificar el extracto y permite alcanzar concentraciones de oro muy bajas. Sin embargo, estas técnicas no se pueden utilizar para todas las matrices debido a que existe una lixiviación incompleta del oro cuando están presentes minerales con sulfuros y otras matrices complejas (Ghosh et al., 2019).

La norma ISO/IEC 17025:2017 ha establecido requisitos claros para la validación de la metodología analítica; sin embargo, esta es una actividad compleja que, además de cumplir con las normas aplicables, debe adaptarse para cumplir con las necesidades de los clientes, características específicas de cada método y realidad del propio laboratorio. Por lo mismo se han creado varias guías en estos temas como la "Guía de Laboratorio para Validación de Métodos y Temas Relacionados" (Barwick et al., 2016). La evaluación de la calidad de una medida se realiza mediante la estimación de la incertidumbre de la misma, por lo que la presentación de un resultado sin reportar su incertidumbre le quita valor a este. El proceso de estimación de la incertidumbre debe considerar las condiciones específicas de cada proceso de medición y adaptarse a este (Bettencourt \& Williams, 2015).

La presente investigación describe el proceso de validación del método para la determinación de oro por ensayo al fuego combinado con espectrofotometría de absorción atómica en sedimentos, rocas, concentrados y relaves, con el objetivo de obtener resultados confiables.

\section{MATERIALES Y MÉTODO}

Para la adaptación y validación de la metodología de análisis de oro en sedimentos, rocas, relaves y concentrados que son de interés geológico, minero y metalúrgico, es importante seleccionar el método de referencia con el que se va a trabajar, los más apropiados para este fin son: ASTM 
E1335 (2017): determinación de oro en lingotes por análisis de ensayo de copelación y SM 3111 (2017): metales por espectrometría de absorción atómica por Ilama.

El proceso inició con la preparación mecánica de muestras, mediante la trituración, molienda, pulverización y cuarteo. Con esto el oro se distribuye equitativamente en la muestra.

A continuación, las muestras pasaron por un proceso de tostación a $700{ }^{\circ} \mathrm{C}$ aproximadamente, en una atmósfera oxidante para eliminar sulfuros y carbonatos. Al material tostado, se le adicionó una carga fundente compuesta por bórax, dióxido de silicio (conocido también como sílice), nitrato de potasio, harina (de trigo) y el óxido de plomo II (PbO), con el fin de fundir la muestra a $1000{ }^{\circ} \mathrm{C}$, para que los metales base formen silicatos y boratos (escoria) y los metales preciosos como el oro y la plata formen una aleación con el plomo (régulo). Se adicionaron gotas de nitrato de plata al $3 \%$, para garantizar que el oro y la plata formen una aleación en relación mayor a 1:3, respectivamente. El régulo de plomo se copeló en un horno a $950{ }^{\circ} \mathrm{C}$, obteniendo la aleación en forma de esfera de oro y plata denominada doré (Cotet et al., 2017).

El doré fue colocado en un recipiente con ácido nítrico diluido hasta obtener botón de coloración negra (disolución de la plata). Este botón fue disuelto con agua regia para obtener el oro presente en la muestra en solución hasta un volumen de aforo de 10 mL ("ASTM E1335, Standard Test Methods for Determination of Gold in Bullion by Fire Assay Cupellation Analysis," 2017).

Para la determinación cuantitativa de oro resultante se utilizó un espectrofotómetro de absorción atómica a la Ilama, el mismo que permite medir la concentración de analito de interés en una solución. El oro se determinó a una longitud de onda de 242,8 nm ("3111 Metals by Flame Atomic Absorption Spectrometry," 2017).

\section{Procedimiento}

El objetivo de la validación es demostrar estadísticamente que el método propuesto para la determinación de oro por ensayo al fuego y lectura por absorción atómica cumple con espe- 
cificaciones de desempeño: intervalo de trabajo, selectividad y especificidad, límite de detección (LD), límite de cuantificación (LC), robustez, linealidad, precisión e incertidumbre (Barwick et al., 2016).

En la Tabla 1, se indican los objetivos de validación que el laboratorio estableció para la determinación de oro por ensayo al fuego.

Tabla 1. Objetivos de validación

\begin{tabular}{ll}
\hline \multicolumn{1}{c}{ PARÁMETRO } & \multicolumn{1}{c}{ OBJETIVO } \\
\hline $\begin{array}{l}\text { Selectividad/ } \\
\text { Especificidad }\end{array}$ & $\begin{array}{l}\text { Evaluar interferencia por } \\
\text { presencia de sulfuros. }\end{array}$ \\
\hline Intervalo de trabajo & $\begin{array}{l}\text { Desde el límite de cuanti- } \\
\text { ficación hasta } 350 \mathrm{mg} / \mathrm{kg} \\
\text { (rango acorde a presen- } \\
\text { cia de oro en muestras } \\
\text { analizadas por el labora- } \\
\text { torio). }\end{array}$ \\
\hline Linealidad/ & $\begin{array}{l}\text { Regresión lineal. R2 } \geq 0,995 \\
\text { Función respuesta }\end{array}$ \\
\hline LD y LC & $\begin{array}{l}\text { Análisis de blancos y con- } \\
\text { firmación experimental. }\end{array}$ \\
\hline $\begin{array}{l}\text { Repetibilidad y } \\
\text { precisión intermedia }\end{array}$ & $\begin{array}{l}\text { ObV } \\
\text { Obtenido de la aplicación } \\
\text { de ecuación de Horwitz. }\end{array}$ \\
\hline Veracidad & $\begin{array}{l}70 \% \text { - 130\% } \\
\text { Criterio establecido por el } \\
\text { método de referencia. }\end{array}$ \\
\hline Incertidumbre & \begin{tabular}{l}
$38 \%$ \\
\hline
\end{tabular}
\end{tabular}

FUENTE: Laboratorio químico IIGE

\section{Límite de detección y límite de cuan-} tificación: Se midieron réplicas de blancos; se utilizó cuarzo fino granular, material que no contiene cantidades detectables del analito. Se calculó el LD y LC, aplicando el promedio de los blancos más 3 y 10 desviaciones estándar, respectivamente (Barwick et al., 2016).

Se comprobaron los resultados teóricos, analizando réplicas de blancos fortificados a las concentraciones teóricas obtenidas del cálculo anterior. Se determinó el coeficiente de variación del porcentaje de recuperación para evaluación de resultados.

Linealidad: La linealidad del método fue corroborada estableciendo un ajuste lineal entre dos variables " $y$ " que corresponde a la señal y " $x$ " a la concentración; este análisis consistió en la construcción de una recta cuyo límite inferior fue el LC y el límite superior el valor en que la curva se desvía y deja de ser lineal.

Este análisis parte de la premisa de que existe una relación lineal entre las dos variables " $x$ " $y$ " $y$ ", por lo que la ecuación general es: 


$$
y=a x+b
$$

Donde " $y$ " es el valor de la variable dependiente, " $\mathrm{x}$ " es el valor de la variable independiente, " $b$ " es la intersección en la ordenada y; $y$ "a" es la pendiente de la recta.

En el desarrollo de este método, se realizaron curvas de calibración a diferentes días y con diferentes analistas y de cada una se obtuvo información de la regresión lineal realizada de acuerdo a la ecuación (1), con esto se comparó y asignó una curva representativa del método, con criterios de aceptación y rechazo para pendiente e intercepto (Barwick et al., 2016).

Selectividad y especificidad: Se evaluó este parámetro analizando muestras con contenido de sulfuros. Se analizaron los resultados comparando los pesos de los régulos obtenidos después del proceso de ensayo al fuego (Barwick et al., 2016).

Robustez: Se establecieron experimentos analizando el material de referencia certificado de concentración 30,87 mg/kg de oro (SQ87 Rocklabs), en condiciones de repetibilidad, cambiando parámetros como: masa de muestras, temperatura de tostación, temperatura de fusión y temperatura de copelación.

\section{Precisión (repetibilidad y precisión} intermedia): Se realizó un diseño experimental para: nivel bajo (límite de cuantificación), nivel medio (153,3 mg Au/kg) y para nivel alto $(353,3$ mg $\mathrm{Au} / \mathrm{kg})$.

Para cada nivel se evaluó precisión intermedia en condiciones de reproducibilidad, variando analistas y días de ensayo; para precisión bajo condiciones de repetibilidad, el ensayo se realizó por un mismo técnico de laboratorio en un mismo equipo analítico.

El análisis de datos se realizó empleando un análisis de varianza de un factor (ANOVA). De los resultados del ANOVA los cuadrados medios de cada una de las componentes de variabilidad, entre los grupos (Mb) y dentro de los grupos (Mw), fueron utilizados para determinar la desviación estándar por repetibilidad (sr) y la desviación estándar por la precisión intermedia (sR) del método. De acuerdo a las siguientes ecuaciones: 


$$
\begin{aligned}
& s r=\sqrt{M_{W}} \\
& s R=\sqrt{s_{r}^{2}+s_{l}^{2}}=\sqrt{M_{W}+\left(\frac{M_{b}-M_{W}}{n}\right)}
\end{aligned}
$$

Donde $\mathrm{n}$ es número de mediciones.

Para saber si existe diferencia significativa entre los datos obtenidos, se utilizó el estadístico de Fisher (prueba F), misma que es obtenida de los resultados del ANOVA, dicha prueba compara varianzas (Bettencourt \& Williams, 2015).

Veracidad: Se midieron réplicas de un material de referencia certificado de concentración de 0,077 mg Au/kg (OxA131) y 37,02 mg Au/kg (AMIS0270). Se evaluó mediante el cálculo del porcentaje de recupera- ción, tomando como referencia el valor certificado del material (Bettencourt \& Williams, 2015).

Incertidumbre: Se aplicó la ley de propagación de la incertidumbre. Para lo cual se siguieron los siguientes pasos:

- Se identificó el mesurando.

- Se identificaron las fuentes que contribuyen a la incertidumbre.

- Se cuantificaron los componentes de la incertidumbre siguiendo los lineamientos de la guía GUM.

- Se calculó la incertidumbre combinada.

- Se calculó la incertidumbre expandida (Bettencourt \& WiIliams, 2015).

\section{RESULTADOS}

Límite de detección y Límite de cuantificación: Experimentalmente se obtuvo LD de 0,013 mg/kg y un LC de $0,033 \mathrm{mg} / \mathrm{kg}$.

Linealidad: Se realizaron pruebas de linealidad en el equipo de absorción atómica para la cuantificación de oro y se determinaron dos curvas de calibración con el fin de conservar la linealidad. En la Tabla 2 se detallan los rangos expresados en concentración de cada curva. 
Tabla 2. Curvas de calibración desarrolladas para la determinación de oro por ensayo al fuego

\begin{tabular}{cccc}
\hline Elemento & $\begin{array}{c}\text { Puntos de } \\
\text { curva }\end{array}$ & $\begin{array}{c}\text { Concentración } \\
(\mathrm{mg} / \mathrm{L}) \\
\text { rango bajo }\end{array}$ & $\begin{array}{c}\text { Concentración } \\
(\mathrm{mg} / \mathrm{L}) \\
\text { ango alto }\end{array}$ \\
\hline \multirow{6}{*}{ Oro } & BLANCO & ---- & ---- \\
& 1 & 0,1 & 2,0 \\
& 2 & 0,2 & 4,0 \\
& 3 & 0,5 & 6,0 \\
& 4 & 1,0 & 8,0 \\
& 5 & 2,0 & 10,0 \\
\hline
\end{tabular}

Se corroboró la linealidad de dichas curvas de calibración mediante una regresión lineal.

Para determinar estadísticamente los intervalos de aceptación de pendiente, intercepto y coeficiente de correlación lineal, se generaron 14 curvas de calibración para rango bajo y 14 curvas de calibración para rango alto, las cuales fueron preparadas de manera independiente y medidas en diferentes días para estudiar la linealidad del método y su comportamiento. De este análisis se obtuvieron dos curvas representativas, con rangos de confianza, indicados en la Tabla 3.

Tabla 3. Curva representativa para la determinación de oro por ensayo al fuego combinado con espectrometría de absorción atómica

\begin{tabular}{ccc}
\hline Parámetros & $\begin{array}{c}\text { Curva representativa } \\
\text { baja }\end{array}$ & $\begin{array}{c}\text { Curva representativa } \\
\text { alta }\end{array}$ \\
\hline $\begin{array}{c}\text { Límite superior } \\
\text { de la pendiente (bs) } \\
\quad \text { Límite inferior }\end{array}$ & 0,070 & 0,062 \\
$\begin{array}{c}\text { de la pendiente (bi) } \\
\text { Límite superior }\end{array}$ & 0,024 & 0,024 \\
$\begin{array}{c}\text { del intercepto (ai) } \\
\text { Límite inferior }\end{array}$ & 0,004 & 0,017 \\
$\begin{array}{c}\text { del intercepto (ai) } \\
r^{2} \text { mínimo obtenido }\end{array}$ & $-0,002$ & $-0,003$ \\
\hline
\end{tabular}


Selectividad y especificidad: Previo a la ejecución de la validación, el análisis de interferencias es primordial; un interferente identificado que afecta directamente a la cuantificación de oro en muestras geológicas, mineras y metalúrgicas son los sulfuros. Se determinó que someter a la muestra a una tostación a temperaturas entre $650-750{ }^{\circ} \mathrm{C}$ antes del proceso de ensayo al fuego elimina el azufre presente.
En la Tabla 4 se observan los resultados de muestras con alto contenido de sulfuros que fueron procesadas con y sin tostación en el proceso de ensayo al fuego; se observó que el doré no se forma correctamente en presencia del interferente si no se trata previamente y existe una pérdida de peso del doré.

Tabla 4. Resultados análisis de interferencias

(evaluación presencia de sulfuros con tratamiento térmico)

\begin{tabular}{cccc}
\hline Muestra & Tratamiento & Peso del régulo $(\mathbf{g})$ & Observaciones \\
\hline 1 & Con tostación & 41,71 & Se obtiene el doré \\
& Sin tostación & 31,87 & No se obtuvo el doré \\
\hline 2 & Con tostación & 41,69 & Se obtiene el doré \\
& Sin tostación & 7,14 & No se obtuvo el doré \\
\hline
\end{tabular}

Robustez: Se evaluó este parámetro mediante el desarrollo de dos experimentos, utilizando material de referencia certificado de concentración 30,87 mg /kg de oro (SQ87 Rocklabs). En el experimento uno, se varió la masa de la muestra en $10 \%$, dejando constantes la temperatura de tostación a $700{ }^{\circ} \mathrm{C}$, fusión $1000{ }^{\circ} \mathrm{C}$ y copelación a $950{ }^{\circ} \mathrm{C}$. En el segundo experimento, se varió la temperatura de tostación, fusión y copelación en aproximadamente $20{ }^{\circ} \mathrm{C}$, dejando la masa de la muestra constante a $124 \mathrm{~g}$. Se realizó la comparación de las medias experimentales, obteniendo que los datos de concentración de oro de las muestras son homogéneos entre sí.

\section{Repetibilidad y precisión intermedia:}

El desarrollo de la repetibilidad y precisión intermedia, se realizó en dos días diferentes y por dos analistas diferentes, se estableció número de ré- 
plicas de $\mathrm{n}=6$, en tres niveles de concentración de oro:, 0,033 mg/kg, $153,3 \mathrm{mg} / \mathrm{kg}$ y $353,3 \mathrm{mg} / \mathrm{kg}$, rango bajo, medio y alto respectivamente. Adicionalmente, para evaluar el efecto matriz se realizó el mismo análisis en muestras de rocas, sedimentos, relaves y concentrados de procesos metalúrgicos. Obteniendo los resultados mediante un análisis de varianza ANOVA, que se presentan en la Tabla 5.

\section{Tabla 5. Resultados repetibilidad ( $r$ ), y precisión intermedia ( $R$ ) obtenidos del ANOVA}

\begin{tabular}{|c|c|c|c|c|}
\hline $\begin{array}{l}\text { Rangos y } \\
\text { matrices }\end{array}$ & $\begin{array}{l}\text { Prueba F } \\
\text { calculado }\end{array}$ & $\begin{array}{l}\text { Prueba F } \\
\text { crítico }\end{array}$ & $\% \mathrm{Cvr}$ & $\% C v R$ \\
\hline $\begin{array}{l}\text { Rango bajo } \\
0,033 \mathrm{mg} / \mathrm{kg}\end{array}$ & 1,071 & 4,301 & 8,596 & 8,622 \\
\hline $\begin{array}{l}\text { Rango medio } \\
153,300 \mathrm{mg} / \mathrm{kg}\end{array}$ & 0,038 & 4,301 & 3,210 & 3,210 \\
\hline $\begin{array}{l}\text { Rango alto } \\
353,3 \mathrm{mg} / \mathrm{kg}\end{array}$ & 3,249 & 4,301 & 5,980 & 6,517 \\
\hline $\begin{array}{l}\text { Sedimento } \\
0,033 \mathrm{mg} / \mathrm{kg}\end{array}$ & 0,378 & 4,600 & 18,273 & 18,273 \\
\hline $\begin{array}{l}\text { Relave } \\
0,350 \mathrm{mg} / \mathrm{kg}\end{array}$ & 3,070 & 4,301 & 10,692 & 10,692 \\
\hline $\begin{array}{l}\text { Roca } \\
4,200 \mathrm{mg} / \mathrm{kg}\end{array}$ & 0,052 & 4,301 & 9,201 & 9,201 \\
\hline $\begin{array}{l}\text { Concentrado } \\
50 \mathrm{mg} / \mathrm{kg}\end{array}$ & 5,706 & 4,301 & 4,604 & 4,604 \\
\hline Criterios & \multicolumn{2}{|c|}{ Fcal < Fcrit } & \multicolumn{2}{|c|}{$\% \mathrm{CV}<19 \%$} \\
\hline
\end{tabular}

Se obtuvo un coeficiente de variación $(\% \mathrm{CV})$ por debajo del objetivo planteado para todos los niveles y muestras matriz; además, el F calculado es menor al F crítico por lo que los resultados no difieren entre sí, consecuentemente, el método es preciso. Sin embargo, únicamente en el análisis de muestras de concentrados, el $\mathrm{F}$ cal es mayor al F crítico, existiendo diferencias significativas en los resultados, es decir no son homogéneos entre sí, esto puede atribuirse a errores sistemáticos en la medición como es el error humano. 
Veracidad: Se determinó ensayando varias veces un mismo material de referencia certificado, se calculó el porcentaje de recuperación promedio, los resultados se detallan en la Tabla 6.

Tabla 6. Resultados de veracidad

\begin{tabular}{ccc}
\hline $\begin{array}{c}\text { Parámetros } \\
\text { estadísticos }\end{array}$ & $\begin{array}{c}\text { MRC } \\
\text { OxA131 } \\
(0,077 \mathrm{mg} / \mathrm{kg})\end{array}$ & $\begin{array}{c}\text { MRC } \\
\text { AMIS0270 } \\
(37,020 \mathrm{mg} / \mathrm{kg})\end{array}$ \\
\hline Promedio & 0,076 & 34,775 \\
\hline Desviación & 0,003 & 1,590 \\
\hline $\begin{array}{c}\text { \% recuperación } \\
\text { promedio }\end{array}$ & & \\
\begin{tabular}{c} 
(criterio 70-130 \%) \\
\hline
\end{tabular} & 98,918 & 93,936 \\
\hline
\end{tabular}

Como resultado se obtuvo que los dos cumplen con el objetivo de validación establecido.

Incertidumbre: Se identificaron todas las fuentes de aportación para la incertidumbre, utilizando un diagrama de causa-efecto (diagrama Ishikawa).

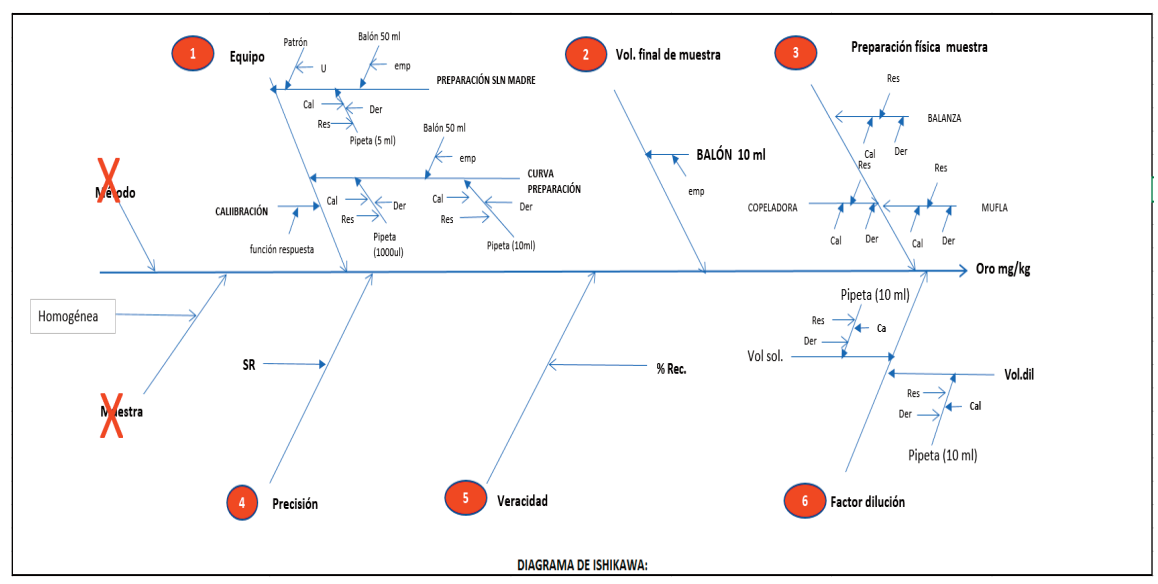

Figura 1. Diagrama causa efecto ejemplo. FUENTE: Laboratorio Químico IIGE

Utilizando la ley de propagación de incertidumbre, se obtuvieron los re- sultados presentados en las Tablas 7 y 8 . 
Tabla 7. Resultados de incertidumbre en fortificaciones (criterio $38 \%$ )

\begin{tabular}{cccc}
\hline Parámetro & $\begin{array}{c}\text { Rango bajo } \\
\mathbf{0 , 0 3 3} \mathrm{mg} / \mathrm{kg}\end{array}$ & $\begin{array}{c}\text { Rango medio } \\
153,300 \mathrm{mg} / \mathrm{kg}\end{array}$ & $\begin{array}{c}\text { Rango alto } \\
353,300 \mathrm{mg} / \mathrm{kg}\end{array}$ \\
\hline $\mathrm{U}(\mathrm{K}=2) \%$ & 19,160 & 12,254 & 17,963 \\
\hline
\end{tabular}

Tabla 8. Resultados de incertidumbre en muestras (criterio $38 \%$ )

\begin{tabular}{ccccc}
\hline Parámetro & $\begin{array}{c}\text { Sedimento } \\
\mathbf{0 , 1 0 4} \mathbf{~ m g} / \mathbf{k g}\end{array}$ & $\begin{array}{c}\text { Relave } \\
\mathbf{0 , 3 5 0} \mathbf{~ m g} / \mathbf{k g}\end{array}$ & $\begin{array}{c}\text { Roca } \\
\mathbf{6 , 1 7 0} \mathbf{~ m g} / \mathbf{k g}\end{array}$ & $\begin{array}{c}\text { Concentrado } \\
\mathbf{1 4 8 , 9 5 4} \mathbf{~ m g} / \mathbf{k g}\end{array}$ \\
\hline$U(\mathrm{~K}=2) \%$ & 27,557 & 22,389 & 14,942 & 12,967 \\
\hline
\end{tabular}

La incertidumbre aportada en cada caso, cumplió con el criterio de aceptación fijado por el laboratorio.

Ensayo de aptitud: Para evaluar el desempeño en la realización del ensayo, en agosto del 2019 se participó en una ronda de ensayo de aptitud internacional, distribuida por LQSI (Laboratory quality services international), ubicado en Estados Unidos de América. Dicha institución envió dos muestras de prueba, las cuales fueron analizadas, tal cual una muestra cualquiera, los resultados de la ronda se detallan en la Tabla 9.

Tabla 9. Resultados de ensayo de aptitud

\begin{tabular}{ccccc}
\hline Muestra & $\begin{array}{c}\text { Resultado } \\
\text { laboratorio } \\
(\mathrm{mg} / \mathrm{kg})\end{array}$ & $\begin{array}{c}\text { Resultado } \\
\text { LQI } \\
(\mathrm{mg} / \mathrm{kg})\end{array}$ & z score & $\begin{array}{c}\text { Criterio de } \\
\text { evaluación } \\
\text { estadístico }\end{array}$ \\
\hline rango bajo & 0,096 & 0,086 & 0,81 & $\begin{array}{r}|\mathrm{Z}| \leq 2-> \\
\text { Satisfactorio } \\
2<|\mathrm{Z}| \leq 3->\end{array}$ \\
\hline rango alto & 5,50 & 5,64 & $-0,56$ & $\begin{array}{r}\text { Alerta } \\
|\mathrm{Z}|>3 \\
\text { Insatisfactorio }\end{array}$ \\
\hline
\end{tabular}

En las dos muestras se obtiene un z score $\leq 2$, cuyo resultado es satisfactorio. 


\section{DISCUSIÓN}

El estudio fue determinado para diferentes matrices, teniendo así concentraciones de oro muy variadas entre cada una de ellas. Por lo tanto, se vio la necesidad de realizar dos curvas de calibración en dos rangos, de manera de disminuir el error por una linealización en un rango tan amplio.

Los resultados obtenidos respecto al tamaño de partícula usado en el ensayo $(<0,075 \mathrm{~mm}$ ) están respaldados, por el estudio de Osman (2018), en el cual se indica que a menor tamaño de grano la recuperación de oro incrementa, por lo que es recomendable trabajar con este tipo de tamaño. Los resultados respecto al tamaño de grano se ven reflejados en los ensayos de repetitividad.

Las principales interferencias (sulfuros), son eliminadas mediante la aplicación del tratamiento térmico de tostación. Luego de la evaluación del método con distintas matrices se concluye que es adecuado para todas ellas. Existen formulaciones para evitar el proceso de tostación que consiste en modificar la cantidad de los fundentes, sin embargo, debido a la variedad de muestras con contenido diferente de sulfuros, el laboratorio considera aplicar tostación.

Mediante el proceso de validación se determinó que el rango de cuantificación de este método fue de 0,033 a 353,3 mg/kg. Determinación de concentraciones bajas son útiles para estudios de prospección de oro. Determinación de concentraciones altas son útiles para estudiar presencia de vetas de oro o evaluar la eficiencia de procesos metalúrgicos de concentración de oro.

EI LD para este método fue de 0,013 $\mathrm{mg} / \mathrm{kg}$ para el laboratorio químico del IIGE y fue contrastado con rangos de laboratorios comerciales como MSALABS, ALS LABS, Bureau Veritas Metals, Minerals \& Enviromental LABS, entre otros que van desde $0,005 \mathrm{mg} / \mathrm{kg}$, por lo cual es un valor congruente.

Del análisis de varianza ANOVA de repetibilidad y reproducibilidad, se determinó que existió mayor variabilidad, en la matriz de concentrados, lo cual se ve reflejado también en la 
alta incertidumbre que tiene esta matriz en comparación con las otras. Para asegurar los resultados reporta- dos, el laboratorio utiliza el valor de la incertidumbre más alta para abarcar todas las matrices.

\section{CONCLUSIÓN}

El método para determinación de oro por ensayo al fuego evaluado es considerado un método selectivo, lineal y veraz en el intervalo de trabajo de $0,033 \mathrm{mg} / \mathrm{kg}$ a $353,3 \mathrm{mg} / \mathrm{kg}$, ya que cumplió con parámetros de validación fijados. Los siguientes son los parámetros que cumple el método: lectura de blancos no excede al LC; curva de calibración con un $r^{2} \geq$ 0,998; valores de los MRC y fortificaciones dentro del rango de 80,67\% a 129,70 \% de recuperación; el análisis de repetibilidad y precisión intermedia el \% CV menor a 18,72 \%. Cumpliendo con estos valores se puede asegurar que el método cuenta con una incertidumbre de medición $\mathrm{U}(\mathrm{k}=2)$ de $27,6 \%$.

El método evaluado para determinación de oro por ensayo al fuego presenta interferencias cuando las muestras contienen sulfuros, provo- cando fundiciones no homogéneas y dorés de menor tamaño; es decir que afecta directamente a la recuperación del oro, lo que se evidenció en el estudio de selectividad y especificidad, concluyendo así que es importante que las muestras sean tratadas previamente mediante un proceso de tostación.

Mediante la participación del ensayo de aptitude se corrobora que el método implementado y validado es correcto, obteniendo resultados satisfactorios con z scores menores a 2.

Los resultados de la validación, respaldan los resultados del laboratorio químico del IIGE, por lo cual, los entes de control minero que utilicen este servicio, pueden hacer uso de los datos para tomar las acciones debidas en el ámbito de su competencia. 


\section{LISTA DE REFERENCIAS}

3111 Metals by Flame Atomic Absorption Spectrometry. (2017). Standard Methods for the Examination of Water and Wastewater (23ND Ed), 1-10. https://www.standardmethods.org/doi/abs/10.2105/SMWW.2882.043

ASTM E1335, Standard Test Methods for Determination of Gold in Bullion by Fire Assay Cupellation Analysis. (2017). In ASTM International (pp. 1-7). https://doi.org/10. 1520/E1335-08R17.2

Balaram, V., Mathur, R., Satyanarayanan, M., Sawant, S. S., Roy, P., Subramanyam, K. S. V., Kamala, C. T., Anjaiah, K. V., Ramesh, S. L., \& Dasaram, B. (2012). A Rapid Method for the Determination of Gold in Rocks, Ores and Other Geological Materials by F-AAS and GF-AAS After Separation and Preconcentration by DIBK Extraction for Prospecting Studies. MAPAN, 27(2), 87-95. https://doi.org/10. 1007/s12647-012-0012-2

Barwick, V., Morillas, P., Ellison, S., Engman, J., Gjengedal, E., Oxenbøll, U., Magnusson, B., Müller, H., Patriarca, M., Pohl, B., Robouch, P., Sibbesen, L., Theodorsson, E., Vanstapel, F., Vercruysse, I., Yilmaz, A., \& Yolci, Ö. P. (2016). La Adecuación al Uso de los Métodos Analíticos. Una guía de laboratorio para validación de métodos y temas relacionados (1. ed.). Eurolab España. https://eurachem.org/images/stories/Guides/pdf/MV_guide_2nd_ed_ES.pdf

Bettencourt, R., \& Williams, A. (2015). Eurachem/CITAC Guide: Setting and Using Target Uncertainty in Chemical Measurement, (1. ed.).

Cotet, G. B., Balgiu, B. A., \& Zaleschi (Negrea), V. - C. (2017). Assessment procedure for the soft skills requested by Industry 4.0. MATEC Web of Conferences, 121, 07005. https://doi.org/10.1051/matecconf/201712107005

Ghosh, P., Mandal, H., Sirisha, B., Sen, U., Goswami, S., \& Kiran, N. V. R. (2019). A Modified Acid Digestion Method for Analysis of Gold in Geological Samples: A Comparative Study. MAPAN, 34(4), 551-558. https://doi.org/10.1007/s12647-01900326-8

King, H. M. (2019). Geoscience news and information. 
Laird, J. (2014). EARTH MATERIALS: Introduction to Mineralogy and Petrology. American Mineralogist, 99(2-3), 556-556. https://doi.org/10.2138/am.2014.630

Liu, Y., Wan, B., \& Xue, D. (2019). Sample Digestion and Combined Preconcentration Methods for the Determination of Ultra-Low Gold Levels in Rocks. Molecules, 24(9), 1778. https://doi.org/10.3390/molecules24091778

Osman Ali Salih, A. (2018). Determination the Effective of Grain Size on Gold Recovery by Integral of Sieve Analysis and Atomic Absorption Spectroscopy Methods. Science Journal of Analytical Chemistry, 6(2), 12. https://doi.org/10.11648/j.sjac. 20180602.11

Rodríguez-Rodríguez, Y., Rivera-Borroto, O. M., García-de la Vega, J. M., Basilio-de la Torre, J., Valentín-Rodríguez, I., \& Pino-Rivero, L. (2018). Determination of Gold in Geological Samples Combining the Fire Assay and Ultraviolet Visible Spectrophotometry Techniques. Academia Journal of Scientific Research, 6(1), 027-033. https://doi.org/10.15413/ajsr.2017.0112

Santos-Munguía, P. C., Nava-Alonso, F., Rodríguez-Chávez, V. M., \& Alonso-González, O. (2019). Hidden gold in fire assay of gold telluride ores. Minerals Engineering, 141(June), 105844. https://doi.org/10.1016/j.mineng.2019.105844

Wang, Y., Baker, L. A., \& Brindle, I. D. (2016). Determination of gold and silver in geological samples by focused infrared digestion: A re-investigation of aqua regia digestion. Talanta, 148, 419-426. https://doi.org/10.1016/j.talanta.2015.11.019 